\section{VTE: Antikoagulation mit NWH}

\author{
Krebspatienten haben generell ein höheres Risiko für eine venöse \\ Thromboembolie (VTE). Welche Rolle spielen niedermolekulare \\ Heparine in der Prophylaxe eines VTE-Rezidivs?
}

$\mathrm{N}$ ach der Akutbehandlung einer VTE wird der Patient in der Regel 3-6 Monate fortgesetzt antikoaguliert, bislang v.a. mit Vitamin-K-Antagonisten (VKA). 2011 belegte eine CochraneAnalyse den Nutzen von niedermolekularen Heparinen (NMH) zur Prophylaxe venöser Thromboembolien bei Krebspatienten. In einer weiteren Metaanalyse wurde nun gezeigt, dass das NMH Tinzaparin im Vergleich zu Vitamin-K-Antagonisten gerade für Krebspatienten Vorteile aufweist.

Die französischen Kollegen um Silvy Laporte analysierten fünf randomisierte Studien, in denen VTE-Patienten nach der Akutphase über 3-6 Monate fortgesetzt mit VKA oder dem NMH Tinzaparin behandelt worden waren. Betrachtet wurden die Parameter VTE-Rezidiv, Blutungen und Tod. Der klinische Nutzen wurde definiert als Kombination dieser drei Endpunkte. Insgesamt wurden in den Studien 1.668 Patienten behandelt, 406 (24\%) mit einer Krebserkrankung. 835 hatten Tinzaparin erhalten, 833 einen VKA. Nach Ende der Antikoagulation wurden die Patienten über ein Jahr nachbeobachtet.

Im Behandlungsverlauf war das relative Risiko für eine VTE in der Gesamtgruppe der mit $\mathrm{NMH}$ behandelten Patienten um $15 \%$ niedriger als unter VKA (RR $\quad 0,85 ; \quad 95 \%$-Konfidenzintervall [95\%-KI] 0,55-1,31). Nach einem Jahr waren die Behandlungsergebnisse jedoch vergleichbar (RR 1,05; 95\%-KI 0,76-1,46).

Bei den behandelten Krebspatienten lag die Rückfallrate am Ende der Behandlungsperiode unter VKA bei 11,3\% und die RR-Reduktion durch das NMH bei $38 \%$ (RR 0,62 ; $95 \%$-KI 0,30-1,31; p $=0,21$ ). Der Unterschied erhöhte sich bis zum Ende der einjährigen Nachbeobachtung auf $59 \%$ und war dann signifikant (RR 0,41; $95 \%$-KI 0,21-0,79; $\mathrm{p}=0,008$ ).

Die gewichtete Inzidenz schwerwiegender Blutungen lag nach drei bzw. sechs Behandlungsmonaten in der $\mathrm{Ge}$ samtpopulation, die mit VKA behandelt worden war, bei 2,4\%. Unter NMH-Therapie war das Risiko einer solchen Blutung - wenn auch nicht signifikant - um $38 \%$ geringer.

Im Hinblick auf den klinischen Nutzen insgesamt hatten die mit dem NMH behandelten Patienten einen leichten, wenn auch nicht signifikanten Vorteil dies sowohl in der Gesamtpopulation (RR 0,85; 95\%-KI 0,64-1,13), als auch bei den Krebspatienten (RR 0,85; 95\%-KI 0,58-1,25).

Fazit: Insgesamt, so die Autoren, unterstützt die Analyse die These, dass VTEPatienten von der Behandlung mit dem NMH Tinzaparin profitieren. Auch wenn die Fallzahlen zu niedrig waren, um statistisch signifikante Ergebnisse zu generieren, gehen die Autoren davon aus, dass Tinzaparin für Patienten, die aufgrund von Kontraindikationen keine VKA zur fortgesetzten Antikoagulation erhalten können, eine gute Option darstellt. Dies gelte insbesondere für Krebspatienten, deren Risiko für ein VTE-Rezidiv noch deutlich höher ist als allgemein.

Doris Berger

Laporte $S$ et al. Long-term treatment of venous thromboembolism with tinzaparin compared to vitamin K antagonists: A meta-analysis of 5 randomized trials in non-cancer and cancer patients. Thromb Res. 2012;130(6):853-8.

Kommentar von Prof. Riess: Niedermolekulare Heparine (NMH) sind nach subkutaner Applikation rasch und zuverlässig wirksame Antikoagulanzien. Sie stellen bei Patienten, bei denen eine Therapie mit Vitamin-KAntagonisten (VKA) nicht durchführbar ist, eine sinnvolle Alternative dar.

In nationalen und internationalen Leitlinien wird bei Tumorpatienten grundsätzlich eine Initialtherapie und Sekundärprophylaxe von akuten venösen Thromboembolien mit NMH empfohlen - abweichend von dem Vorgehen bei Nichttumorpatienten, bei denen VKA, bzw. neuerdings auch der Faktor-Xa-Inhibitor Rivaroxaban zur Sekundärprophylaxe angeraten werden. Diese Emp- fehlung basiert bisher auf einer großen Studie mit dem NMH Dalteparin sowie der Metaanalyse unter Einbeziehung auch kleinerer anderer Studien mit unterschiedlichen NMH [Andras A et al. Cochrane Database Syst Rev. 2012;10:CD002001]. Die verfügbaren $\mathrm{NMH}$ unterscheiden sich jedoch u.a. bezüglich Molekulargewichtsverteilung, relativer Antithrombin/Anti-Faktor-Xa-Aktivität und Kumulationsrisiko bei Niereninsuffizienz, sodass die Übertragbarkeit von Studiendaten von einem auf ein anderes NMH zu Recht kritisch hinterfragt werden kann.

Die französische Arbeitsgruppe um Silvy Laporte belegt nun mit ihrer Analyse für Tinzaparin, ein einzelnes NMH, im Vergleich mit VKA an einem gemischten Patientenkollektiv mit venöser Thromboembolie ein zahlenmäßig - wenn auch nicht signifikant - vermindertes Blutungs- sowie Rezidivrisiko. Bei dem Viertel der Patienten mit akuter VTE und Tumorleiden zeigt sich durch Tinzaparin ein vermindertes Rezidivrisiko bei unverändertem Blutungsrisiko in Übereinstimmung mit den Leitlinienempfehlungen. Von Interesse ist darüber hinaus, dass in diese Auswertung verschiedene VKA - nicht nur Warfarin, sondern auch etwa 220 Patienten unter Acenocoumarol - eingingen.

Diese Ergebnisse bestätigen die klinische Wirksamkeit der therapeutischen EinmalGabe von Tinzaparin besonders bei Krebspatienten mit akuter VTE über die Daten der Cancer-LITE-Studie [Akl EA et al. J Exp Clin Cancer Res. 2008;27:21] hinaus. Die Ergebnisse der aktiv rekrutierenden großen prospektiven Phase-III-Studie, in der Tinzaparin im Vergleich zu VKA zur Sekundärprophylaxe an etwa 900 Malignompatienten mit VTE untersucht wird, werden 2014 erwartet und die Evidenzlage wesentlich beeinflussen.

Für die tägliche Praxis bedeutet dies, dass gegenwärtig von den verschiedenen $\mathrm{NMH}$ vorrangig Dalteparin und Tinzaparin über eine solide Datenbasis für die leitliniengemäße Initialbehandlung und Sekundärprophylaxe von Tumorpatienten mit akuter VTE verfügen.

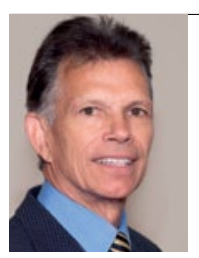

Prof. Dr. Hanno Riess, Medizinische Klinik mit Schwerpunkt Hämatologie, Onkologie und Tumorimmunologie, Charité - Universitätsmedizin Berlin hanno.riess@charite.de 